\begin{tabular}{c} 
International Journal of Engineering \& Technology, 7 (2.7) (2018) 558-564 \\
International Journal of Engineering \& Technology \\
SPC \\
Website $:$ www.sciencepubco.com/index.php/IJET \\
Research Paper \\
\hline
\end{tabular}

\title{
Optimal Scheduling of Micro Grid for Plug-In Electrical Vehicle
}

\author{
K. Vijay Kumar ${ }^{*}$, T. Bharath Kumar² \\ ${ }^{I}$ M.Tech student, EEE Department, Koneru Lakshmaiah Educational Foundation, Vaddeswaram, Guntur (District), A.P, INDIA \\ ${ }^{2}$ Koneru Lakshmaiah Educational Foundation, Vaddeswaram, Guntur (District), A.P, INDIA \\ *Email: vijaykumarkuchipudi143@gmail.com
}

\begin{abstract}
Owing to several advantages of Plug-in Electric Vehicles (PEVs) like less noise, emission less, good efficiency and the reduced cost has attention to the governments, researchers and manufactures in recent time. The Plug-in Electric Vehicle (PEV) plays a vital role in replacement of conventional vehicles in future, because of penetration of renewable energy resources in conventional generation. The modernized of micro grid is happening due to usage of clean energy for EV charging. The cost of electric vehicle charging is challenging issue in the development of plug-in electric vehicle. The coordination between renewable generation and conventional generation is very much needed in near future. The dynamic nature of renewable energy resources causes frequent interrupts in electric vehicle charging. The problem of nonlinear power generation with renewable resources is overcome by electric vehicle battery storage system which enables the EV battery to charge during low demand period and gets discharged into the micro grid during high demand periods. This paper developed an optimal schedule for stationary Plug-in Electric Vehicle charging in operation with micro grid. The obtained optimal schedule provides balance between active and reactive power in generation and load as well. The integration of renewable energy resources is achieved through solar, wind in Vehicle-2-Grid (V2G) approach which is used to safeguard to renewable energy resources by store additional energy produced during peak load period and feeding back to the micro grid during low load period. As a result the stable operation of the micro grid and EV charging with low cost is achieved in this paper.
\end{abstract}

Keywords: Charging and Discharging; Convex optimization; Plug-in Electric Vehicle (PEV); Vehicle-To-Grid (V2G).

\section{Nomenclature}

K Interval set,

RE Renewable energy sources,

N Set of electric vehicles (EVs),

$b_{m j} \quad$ EV charging power in interval $j$,

$T_{m} \quad$ EV charging period,

$\lambda \quad$ Length of an time interval,

$A_{o} \quad$ Real time price model intercept,

$A_{1} \quad$ Slope of the real time price model,

$A_{2} \quad$ Operation, Investment and Maintenance parameter of wind generation,

$A_{3} \quad$ Operation, Investment and Maintenance parameter of solar generation,

$N^{C H G}$ Only EV charging set,

$N^{V 2 G}$ Vehicle-2-grid (V2G) EV set,

$P_{w} \quad$ Wind power generation,

$P_{s} \quad$ Solar power generation,

$L_{l}^{b F} \quad$ Forecasted base load in interval $j$,

$E_{m}^{i n i} \quad$ Initial EV battery energy,

$E_{m}^{c a p} \quad$ Capacity of EV battery,
$P^{\max } \quad$ Maximum EV charging power,

$y_{j} \quad$ EVs charging load in interval $j$,

$p_{j} \quad$ Total load in interval $j$,

$\gamma_{m} \quad$ Final EV energy ratio,

$L_{j}^{b} \quad$ Real base load in interval $j$,

$C_{j} \quad$ Each EV charging cost in interval $j$,

$W_{K}^{(j)} \quad$ Sliding window at the staring interval $j$,

$H_{K}^{(j)} \quad$ Ongoing set of EV at the starting interval $j$,

G Charging-interval matrix,

$T_{g s o} \quad$ Total charging cost for global optimal scheduling scheme,

$T_{l s o}^{(j)} \quad$ Total charging cost for local optimal scheduling scheme,

$\beta, \eta \quad$ Model parameters for battery lifetime cost reduction.

\section{Introduction}

The conventional vehicles are creating more air pollution and harmful gases in metropolitan regions. Fuel-based conventional fuel based vehicles deliver a large amount of air pollution in each and every single year. For example, in the United States of America, approximately $28 \%$ of global warming is initiated by fuel-based vehicles i.e. trucks, buses and cars [1]. Not only does conventional fuel based internal combustion engines contribute to 
ecological pollution but they use a huge amount of fuel for conventional vehicles. Electricity generation and transportation of conventional vehicles are considering the main contributing factor to air pollution and as well as global warming. The daily usages of fuel for conventional vehicles are nearly 430 million gallons in the United States of America [2]. To minimise the usage of fuel for conventional vehicles and air pollution then we take new technology in vehicles and it is electric vehicle technology. This technology is the essential gifted solution to challenges this issue (usage of fuel and air pollution). These electric vehicle resolution information are leads conventional vehicles manufactures to move and spend money in the electric vehicles production [29].

In the late 1800s, the marketable electric vehicle was launched in New York City [3]. Later, conventional fuel based vehicles are replacing the new automobile manufacturers have entered in the market for plug-in electric vehicle transport. Currently, PEV is reducing carbon dioxide $\left(\mathrm{CO}_{2}\right)$ emission by around $25 \%$ compared to conventional gasoline vehicles [4]. In 2020, EV's sales are growing about $60 \%$ worldwide. In the Indian scenario, the government should provide the initial user base and demand to help technologies cross the valley. The government also gives the importance of the electric vehicles to install EV infrastructure and new governments at all levels. Although some organizations in India are working hard to help India switch to electric transportation, utilizing what Tesla and other EV manufacturers have to offer will save a lot of time. Manufacturers are the interest of EV's, Chevy Volt, Tesla, Nissan, GM, Honda, BMW Mercedes, etc. has held these EVs in all over the world. Plug-in Electric Vehicles (PEV) is considered as a new generation of vehicles. In 2020 , approximately $50 \%$ of new electric vehicles are expected in the international market [6],[28]. Furthermore, various methods have been proposed and are implemented to guarantee optimum power transfer in Vehicle-2-Grid (V2G) system to increase the benefits of V2G [8],[20].

In [9], Yifeng He et al. recommend the global and local optimal scheduling problem for EVs charging and discharging as follows.

The global scheduling optimal problem objective function is to optimise the total cost for all EVs charging during a day. The global optimal scheduling scheme is unfeasible subsequently it needs more information for the arrival times and upcoming base loads of the EV's and charging intervals of the EV's in the upcoming time of the day. The local scheduling optimal problem the EV's in the local region. The local optimal schedule model is very relevant for the EV's charging and discharging and it can capably handle a bulky number of EV's and dynamic of the EVs arrival The local optimal schedule model is less than the global optimal scheduling scheme.

Since the EV's market growing rapidly, penetration of the EV's investigation is the major challenge. The micro grid also facing an over loading problem at peak load conditions [21]-[27]. In both global and local scheduling problem the Plug-in Electric Vehicle charging cost is high [15]-[20]. The lifetime of the EV battery also decreased due to frequent charging and discharging period. To overcome these problems to integrate the renewable energy sources having more advantages[33-38].

The proposed scheme is to integrate the plug-in electric vehicle (PEV) to deal with the environmental pollution and global energy crisis, it's necessary to the integration of the renewable energy generation like solar power generation and wind power generation into the existing grid. Additionally, due to the increasing the fuel cost, driving PEV yearly saves a lot fuel cost. More number of EV's were considered to be adjustable generators, so that they can provide energy to minimise both the emission and cost. Nowadays increasing the amount of electricity is produced by the help of renewable energy sources like wind and solar. This renewable energy sources electricity can be consumed by EV which is a great share of the total emissions. Mainly, in Plug-in Electric Vehicles (PEVs) for green transportation has more attracted the customer. Because of the irregular nature for renewable energy sources like solar and wind to face a significant integration of renewable ener- gy sources power poses a challenge on the micro grid in both steady state as well as the transient state. To minimise the EVs charging cost integration the plug-in electric vehicle plays a key role in load scheduling.

The rest of the paper is planned as follows; section II briefly provide the Parameters of solar and wind generation, section III global optimal scheduling, section IV local optimal scheduling, section $\mathrm{V}$ battery lifetime cost reduction section VI simulation results and section VII conclusion.

\section{Solar and Wind parameters}

A micro grid consisting of Wind Generation (WG), Photo Voltaic $(\mathrm{PV})$ generation and load demand is considered. As the renewable energy act, the solar power generation and wind power generation essential to adopt when it is generating. After including the solar power generation and wind power generation cost the total charging cost will be minimised. The optimal charging is executed by exhausting in the Matlab program. The 24 hours real base load is assumed as shown in Table.1.

Table 1: Real Baseload for 24 hours

\begin{tabular}{|c|c|c|c|}
\hline \multirow{2}{*}{ Hours } & Power $(\mathbf{K W})$ & Hours & Power $(\mathbf{K W})$ \\
\hline 1 & 1068 & 13 & 1476 \\
\hline 2 & 1003 & 14 & 1498 \\
\hline 3 & 936 & 15 & 1504 \\
\hline 4 & 898 & 16 & 1502 \\
\hline 5 & 903 & 17 & 1500 \\
\hline 6 & 920 & 18 & 1493 \\
\hline 7 & 981 & 19 & 1458 \\
\hline 8 & 1079 & 20 & 1373 \\
\hline 9 & 1183 & 21 & 1355 \\
\hline 10 & 1261 & 22 & 1323 \\
\hline 11 & 1349 & 23 & 1230 \\
\hline 12 & 1436 & 24 & 1117 \\
\hline
\end{tabular}

The wind and solar information required for carried out the study of the power generated for 24 hours of a day. The cost function of wind and solar generation is as in (1)[16] and it considers the operation, investment, and maintenance cost of the energy generation. The power generation of renewable energy sources for 24 hours during a day is shown in below Table. 2 .

In the total EVs charging costs for the three methods are mentioned below for the micro grid are compared. The three methods are considered for charging the cost of EVs:

1. Total EV charging cost without renewable energy cost and battery lifetime reduction cost.

2. Total EV charging cost includes battery lifetime reduction cost without renewable energy cost.

3. Total EV charging cost includes both battery lifetime reduction cost and renewable energy cost.

The total renewable energy sources cost is,

$f(R E)=\left(A_{2} \times P_{W}\right)+\left(A_{3} \times P_{S}\right)$

\section{Global optimal scheduling}

In each EV arrival and departure time is assumed in global optimal scheduling scheme. As per the real-time pricing model the global optimal scheduling for the EVs charging and discharging. The optimization problem provides the minimization of total charging cost. 
Table 2: Wind and Solar power generation for 24 hours

\begin{tabular}{|c|c|c|}
\hline Time (Hrs.) & $\begin{array}{c}\text { Wind generation } \\
(\mathbf{k W})\end{array}$ & $\begin{array}{c}\text { Solar generation } \\
(\mathbf{k W})\end{array}$ \\
\hline 1 & 1.7 & 0 \\
\hline 2 & 8.5 & 0 \\
\hline 3 & 9.27 & 0 \\
\hline 4 & 16.66 & 0 \\
\hline 5 & 7.22 & 0 \\
\hline 6 & 4.91 & 0.03 \\
\hline 7 & 14.66 & 6.27 \\
\hline 8 & 26.56 & 16.98 \\
\hline 9 & 20.88 & 24.05 \\
\hline 10 & 17.85 & 39.37 \\
\hline 11 & 12.8 & 7.41 \\
\hline 12 & 18.65 & 3.65 \\
\hline 13 & 14.35 & 31.94 \\
\hline 14 & 10.35 & 26.81 \\
\hline 15 & 8.26 & 10.08 \\
\hline 16 & 13.71 & 5.3 \\
\hline 17 & 3.44 & 4.2 \\
\hline 18 & 1.87 & 2.31 \\
\hline 19 & 0.75 & 0 \\
\hline 20 & 0.17 & 0 \\
\hline 21 & 0.15 & 0 \\
\hline 22 & 0.31 & 0 \\
\hline 23 & 1.07 & 0 \\
\hline 24 & 0.58 & 0 \\
\hline & & \\
\hline
\end{tabular}

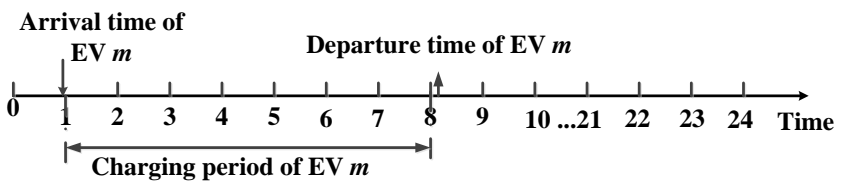

Fig.1: EV charging time interval.

Fig. 1 shows the charging time interval of EV $m$. The EV's battery are charging and discharging throughout a day, it is equally separated in a set of intervals. The set of intervals are signified by $\mathrm{K}$. The length of the charging interval is signified by $\lambda$. To consider the charging power or discharging power in every interim is kep unaffected. The 24 hours in a day is divided in 24 intervals and each interval length is specified as $\lambda=1 \mathrm{~h}$.

The EVs set is execute the charging and discharging in a particular day is signified by N. The set of EV consists of two methods of operation: 1. the set of EVs charging $N^{C H G}$, it contains the charging to the EVs battery only and the battery energy don't supply to the grid and 2. The set EVs to V2G $N^{V 2 G}$, it contains the EVs achieves both battery charging and discharging into the grid. The total EV power in both charging and discharging is $N=N^{C H G}+N^{V 2 G}$. The EV charging power and $\mathrm{EV}$ discharging power of EV $m$ in interim $\mathrm{j}$ is signified by $b_{m j}(\forall m \in N, \forall j \in K)$. The EVs arrival time is signified by $t_{m}^{a r r i v a l}$. The plug-in time of EVs is coupled to the EV charging station. The EVs departure time is signified by $t_{m}^{\text {departure }}$. The time of EVs are going out of EV charging station. The total EV charging period signified by $T_{m}$ this period in this charging and discharging to its EV battery. The connection between the charging/discharging accomplishments, intervals and it describe a Charging-interval matrix $G \subset\{0,1\}^{|N| x|K|}$. If the charging interval of EV are within the region then $G=1$, otherwise $G=0$. The features of $G$ is signified as,

$G_{m j}=\left\{\begin{array}{c}1, \text { if interval jfalls, within the EV charging period } \mathrm{T}_{\mathrm{m}} \\ 0, \text { otherwise }\end{array}\right.$

The EV scheduling is mainly in charging and discharging in a small environmental region. The real-time pricing model, the three assumptions are mainly sorted out in EV charging: 1. the losses between the two nodes are very lesser and unimportant, 2. there is no stoppage in the transmission line, and 3. the baseload $L_{j}^{b}$ remains constant in interval $j$.

The first two assumptions are agreeing us to disregard the spatial difference of electricity price model. In EV optimization is a based on EV charging only sequential difference but not a spatial difference in the price in [11]. The modelling of linear electricity price function in the direct load [11], it is specified as follows:

$f\left(p_{t}\right)=A_{0}+A_{1} \times p_{t}$

Where $A_{0}, A_{1}$ are both in positive real numbers and total load $p_{t}$ at time $t$ and The total load contains of two quantities: 1.The real base load $L_{j}^{b}$, it signifies a load of all electricity feedings in interim $j$ excepting EVs charging and 2.The EV charging load $y_{j}$, this signifies the EVs charging load in interim $j$. The EV charging load in interim $j$ is specified by $y_{j}=\sum_{m \in N} b_{m j} \times G_{m j}$. The total load in interval $j$ is given by $\boldsymbol{p}_{j}=\boldsymbol{L}_{j}^{b}+\boldsymbol{y}_{j}$. To express the charging cost in interim $j$ signified by $C_{j}$. The total sums of cost for clients compensation for charging of their EVs. As per linear pricing model, the EV charging cost is specified by

$$
\begin{aligned}
C_{j} & =\int_{L_{j}^{b}}^{p_{j}}\left(A_{0}+A_{1} \times P_{t}\right) d p_{t} \\
& =\left(A_{0} \times p_{j}+\frac{A_{1}}{2} \times p_{j}^{2}\right)-\left(A_{0} \times L_{j}^{b}+\frac{A_{1}}{2} \times\left(L_{j}^{b}\right)^{2}\right)
\end{aligned}
$$

The total EV charging cost is well-defined as the sum of the EV charging cost. The total amount EV charging cost is specified by

$$
\begin{gathered}
C_{\text {tot }}=\sum_{j \in K} C_{j} \\
=\sum_{j \in K}\left(\left(A_{0} \times p_{j}+\frac{A_{1}}{2} \times p_{j}^{2}\right)-\left(A_{0} \times L_{j}^{b}+\frac{A_{1}}{2} \times\left(L_{j}^{b}\right)^{2}\right)\right)
\end{gathered}
$$

The global optimal scheduling problem is indicated as to optimise the total EVs cost which implements both charging and discharging throughout the day and the total load $p_{j}$ in interval $j$ and the EVs charging power $\boldsymbol{b}_{m j}$.

Mathematically, the global optimal scheduling problem can be expressed as follows:

Minimize $b, p$

$\sum_{j \in K}\left(\left(A_{0} \times p_{j}+\frac{A_{1}}{2} \times p_{j}^{2}\right)-\left(A_{0} \times L_{j}^{b}+\frac{A_{1}}{2} \times\left(L_{j}^{b}\right)^{2}\right)-\left(A_{2} \times P_{W j}\right)-\left(A_{3} \times P_{S j}\right)\right)$

Subject to

$$
\begin{aligned}
& z_{j}=L_{j}^{b}+\sum_{m \in N} b_{m j} \times G_{m j}, \forall j \in K \\
& 0 \leq E_{m}^{i n i}+\sum_{o \in Q^{(j)}} \lambda \times b_{m o} \times G_{m o} \leq E_{m}^{c a p}, \forall m \in N, \forall j \in K \\
& E_{m}^{i n i}+\sum_{j \in Q^{(j)}} \lambda \times b_{m j} \times G_{m j} \geq \gamma_{m} \times E_{m}^{c a p} ;, \forall m \in N \\
& 0 \leq b_{m j} \leq P^{\max }, \forall m \in N^{C H G}, \forall j \in K
\end{aligned}
$$




$$
-P^{\max } \leq b_{m j} \leq P^{\max }, \forall m \in N^{V 2 G}, \forall j \in K
$$

In the above equations, the global optimization problem (6-11), the main objective function of (6) is it minimises the total EVs cost and accomplish charging and discharging in every day. The constraints (7) signify the association among the total load and individual EV charging energy. The constraints (8), (9) are the instant value of EV battery energy and the final value EV battery energy and the constraints (10), (11) are the limitations of the maximum power of EV charging and EV discharging through the grid.

The global optimal scheduling scheme is unrealistic owing to the succeeding causes. First one, all the EVs arrive in the future are unidentified at the existing instant. The next one, the future base load during the day is unidentified at the existing instant. The final one, if a bulky number of EVs are scheduling is the main problem in global scheduling. It may be overrun to neglect this problem scalable centralized scheduling scheme is more important.

\section{Local optimal scheduling}

The global controlling area is divided into groups (LC1, LC2... LCn). In area wise to control the EV vehicle is called Local Controller (LC). Each group charging interval are different and these intervals are within arrival and departure time intervals. The local optimal scheduling scheme is shown in below fig. 3 .

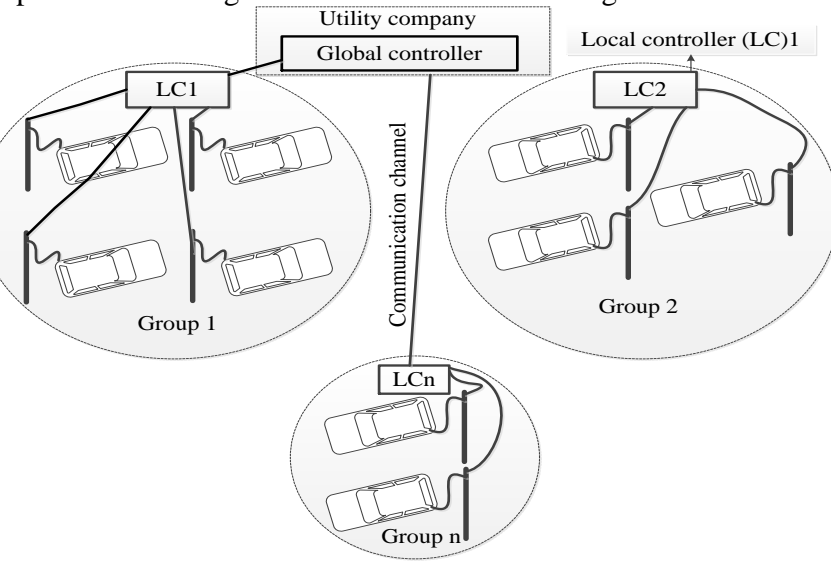

A. Controlling for the local optimal scheduling problem.

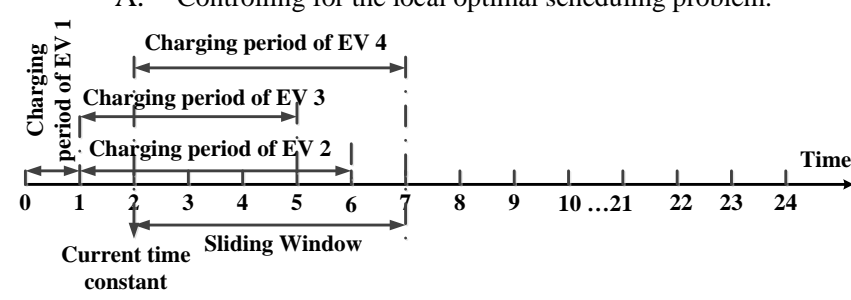

B. set of EV ongoing sliding window

Fig. 2: Ongoing set of EVs by using the sliding window in time scaling of the local optimal scheduling scheme.

The group set is specified as D. subsequently every local controller achieves schedule individually. The local controller doesn't identify the future arrival time of EVs in a group. Therefore, this proposed scheme is to keep informed the EVs charging power at the beginning of every interim with the help of a sliding window. To determine the ongoing of existing EV set $\boldsymbol{H}_{K}^{(j)}$ and the sliding window of the existing set $W_{K}^{(j)}$. The EV $m$ performs both charging and discharging actions throughout its charging period. From the establishment of interim $j$, it describes a charging-interval matrix. If the charging interval of EV are within the existing EV then $G=1$, otherwise $G=0$. The features of $G$ is signified as,
$G_{m l}^{(j)}=\left\{\begin{array}{c}1, \text { if intervalj falls within } \mathrm{H}_{\mathrm{K}}^{(j)} \text { and } \\ \text { with the EV chargingtime of EV } \\ 0, \text { otherwise }\end{array}\right.$

The real base loads present in sliding window $W_{K}^{(j)}$ can be predicted by using time-series methods, regression methods and similar day approach [10]. The similar-day approach [10] is assumed for prediction of the real base load and which is signified by $L_{l}^{b F}$.

Mathematically, the local optimal problem can be conveyed as follows.

Minimize b, $p$

$$
\sum_{l \in W_{k}^{(l)}}\left(\left(A_{0} \times p_{l}+\frac{A_{1}}{2} \times p_{l}^{2}\right)-\left(A_{0} \times L_{l}^{b}+\frac{A_{1}}{2} \times\left(L_{l}^{b F}\right)^{2}\right)-\left(A_{2} \times P_{W l}\right)-\left(A_{3} \times P_{S l}\right)\right)
$$

Subject to

$$
\begin{aligned}
& z_{l}=L_{l}^{b F}+\sum_{m \in H_{K}^{(j)}} b_{m l} \times G_{m l}^{(j)}, l \in W_{K}^{(j)} \\
& 0 \leq E_{m}^{i n i}+\sum_{u \in Q^{(j)}} \lambda \times b_{m u} \times G_{m u}^{(j)} \leq E_{m}^{c a p}, m \in H_{K}^{(j)}, l \in W_{K}^{(j)} \\
& E_{m}^{(j) i n i}+\sum_{l \in W_{K}^{(j)}} \lambda \times b_{m l} \times G_{m l} \geq \gamma_{m} \times E_{m}^{c a p} ;, \forall m \in H_{K}^{(j)} \\
& 0 \leq b_{m l} \leq P^{\max }, \forall m \in H_{K}^{(l) V 2 G}, \forall l \in W_{K}^{(j)} \\
& -P^{\max } \leq b_{m l} \leq P^{\max }, \forall m \in H_{K}^{(l) V 2 G}, \forall l \in W_{K}^{(j)}
\end{aligned}
$$

In the above equations, the local scheduling problem (13-18), in the main objective function (13) to be optimised the total EVs cost and it execute charging and discharging throughout the day. The constraint (14) signifies the relationship between the total load in the interval and the individual EV charging power. The constraints (15), (16) are the instant energy of EV battery constraints and final energy of EV battery constraints. The constraints (17), (18) are the limitations of EV charging and EV discharging through the grid.

\section{Battery lifetime cost reduction}

In each EV battery lifetime may be decreased due to everyday charging and discharging. The modeling of battery lifetime reduction cost for EV $m$, signified by $\psi_{m}$. The two major cost arrangements are more important in EV battery lifetime reduction. Therefore, the amount of charging cost $\psi_{m}^{A}$ and the amount of fluctuation for charging and discharging cost component $\psi_{m}^{F}$ between two sequential intervals. Basically, the EV battery cost component $\psi_{m}^{A}$ is contingent on the total sum of EVs charging and discharging power in every day and it is specified as,

$$
\psi_{m}^{A}=\sum_{j \in K} \beta \times b_{m j}^{2}
$$

Where $b_{m j}$ the charging power of EV $m$ and $\beta$ is the model constraint. Similarly, the amount of fluctuation for charging and discharging cost component $\psi_{m}^{F}$ in every day and it is specified as,

$\psi_{m}^{F}=\sum_{j=2}^{|K|} \eta \times\left(b_{m j}-b_{m(j-1)}\right)^{2}$

The total EVs cost of battery lifetime reduction in throughout the day is specified as,

$$
\psi=\sum_{m \in N} \psi_{m}=\sum_{m \in N}\left(\psi_{m}^{A}+\psi_{m}^{F}\right)
$$




$$
\psi=\sum_{m \in N}\left(\sum_{j \in K} \beta \times b_{m j}^{2}+\sum_{j=2}^{|K|} \eta \times\left(b_{m j}-b_{m(j-1)}\right)^{2}\right)
$$

The total cost of battery lifetime reduction is adding with the main objective function of the global optimal scheduling scheme is reformed by,

$$
\begin{aligned}
& T_{g s o}=\sum_{j \in K}\left(\left(A_{0} \times p_{j}+\frac{A_{1}}{2} \times p_{j}^{2}\right)-\left(A_{0} \times L_{j}^{b}+\frac{A_{1}}{2} \times\left(L_{j}^{b}\right)^{2}\right)-\left(A_{2} \times P_{W j}\right)-\left(A_{3} \times P_{S j}\right)\right)+ \\
& \sum_{m \in N}\left(\sum_{j \in K} \beta \times b_{m j}^{2}+\sum_{j=2}^{|K|} \eta \times\left(b_{m j}-b_{m(j-1)}\right)^{2}\right)
\end{aligned}
$$

After adding the lifetime cost of the battery the global optimal scheduling objective function $T_{g s o}$ is in equation (22). Similarly, local optimal scheduling objective function $T_{l s o}^{(j)}$. After adding the cost of total battery lifetime reduction, the main objective function of the local optimal scheduling scheme is reformed as

$T_{l s o}^{(j)}=\sum_{l \in W_{k}^{(l)}}\left(\left(A_{0} \times p_{l}+\frac{A_{1}}{2} \times p_{l}^{2}\right)-\left(A_{0} \times L_{l}^{b}+\frac{A_{1}}{2} \times\left(L_{l}^{b F}\right)^{2}\right)-\left(A_{2} \times P_{W l}\right)-\left(A_{3} \times P_{S l}\right)\right)+$

$\sum_{m \in N}\left(\sum_{j \in K} \beta \times b_{m j}^{2}+\sum_{j=2}^{|K|} \eta \times\left(b_{m j}-b_{m(j-1)}\right)^{2}\right)$

Here, the existing ongoing EV set $H_{K}^{(j)}$ and the existing sliding window $W_{K}^{(j)}$. The lifetime cost of EV battery plays a key role in global optimal and local optimal scheduling scheme. The two schemes are solved by using convex optimization method. By utilizing this convex optimization method makes to solve efficiently with the interior point schemes [11].

\section{Simulation results}

The total electric load is taken from microgrid. EV testing for charging and discharging is made throughout the day for 24 hours (from 12:00 am). Eventually, 24 interims are considered breaking each interim with a period of $1 \mathrm{hr}$ [30]. The unit measurement of electricity price is Rupees (₹)/Kwh. The electricity pricing model presented in equation (3). We assume $\boldsymbol{A}_{\mathrm{O}}=1 \mathrm{E}-4$ ₹ $/ \mathrm{kWh} \boldsymbol{A}_{1}=1.2 \mathrm{E}-4$ ₹ $/ \mathrm{kWh} / \mathrm{kW}, \boldsymbol{A}_{2}=0.32$ ₹ $/ \mathrm{kWh}$ and $\boldsymbol{A}_{3}=0.06$ ₹ $/ \mathrm{kWh}$. The electric vehicle battery parameters are based on the terms of the Chrevolet Volt [29]. The capacity of electric vehicle battery charging is $16 \mathrm{kWh}$ and the range in distance up to 64.0KMs [31]. We considering all EVs are in same specifications. The maximum energy at charging period reaches at slightest $90 \%$ of the EV battery capacity. The maximum electric vehicle charging power for all EVs is fixed to $P_{\max }=5.0 \mathrm{~kW}$. The each EV initial energy is equally spread between 0 and $80 \%$ of the battery capacity. The help of CVX tool box to calculate the optimization problems (6-11) and (13-18) in convex programs. [32], [12].

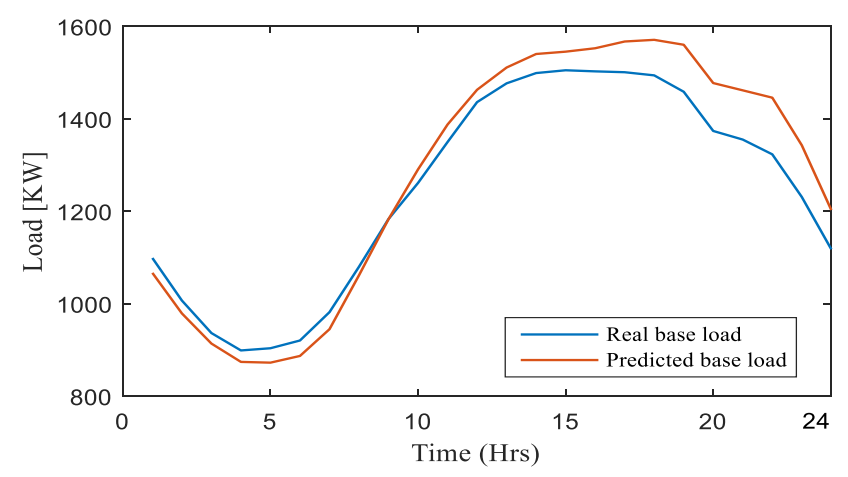

Fig. 3: Comparison between the real and the forecasted base load.

To compare this three optimal scheduling schemes are: 1 . the global optimal scheduling scheme gives an optimal value of the global problem, 2. the local scheduling scheme gives an optimal value of the local problem and 3. The equal allocation scheme is the EV charging/discharging power in interim is allocated based on the succeeding norms: $\mathrm{X}$ ) charge or discharge of EV in an interim is based on the electricity price on the earlier day, and Y) the charging power of the EV is equal in each interval based on absolute value. The total number of EV's are 200. The total EVs are separated into two sub-groups and each sub-group contains 100 EV. All EVs can execute both charging and discharging.

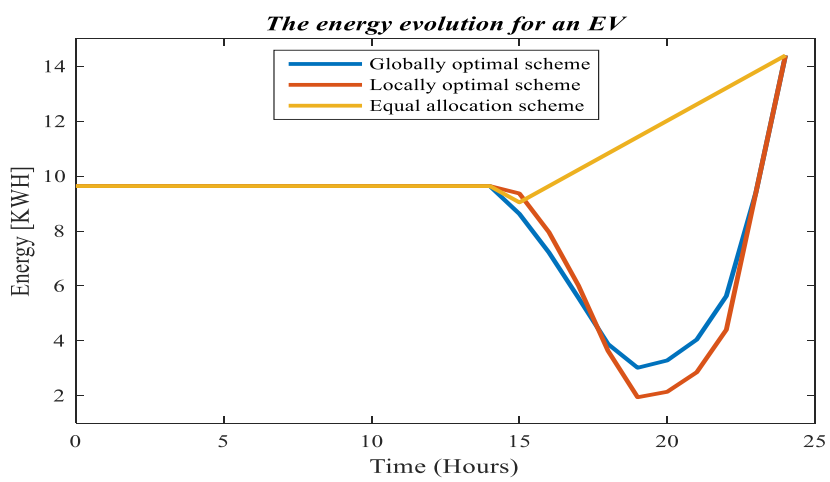

A. Energy

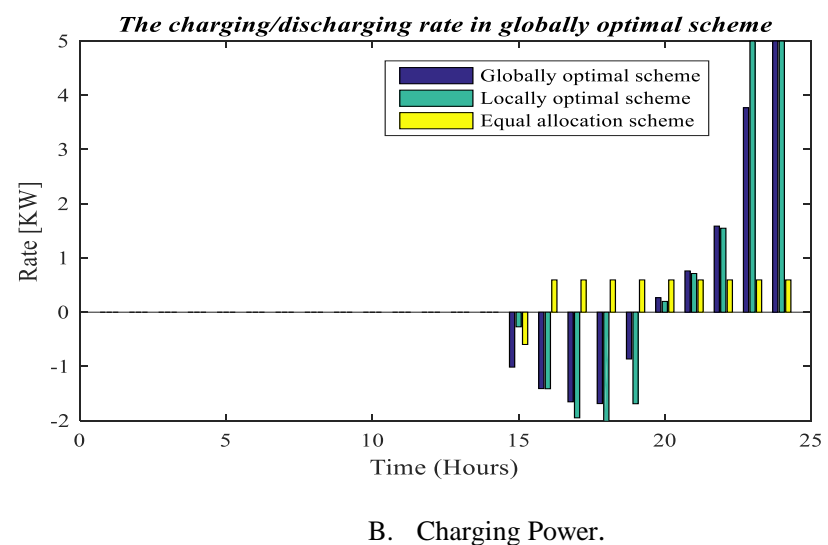

Fig.4: EV 65 charging power and energy in individual interim.

The optimal scheduling of the EV charging power is arbitrary taken EV (e.g., EV 65) in Fig. 4. The charging/discharging period of EV 65 is from interval 15 to 24. In Fig.4. Shows the equal allocation scheme discharges the battery in interval 15, then EV next interval onwards it's charging in equally in each interval. Similarly, the global and local optimal scheduling schemes by solve optimal scheduling problems (5-10) and (11-16) respectively. In order to the reasonable association, the total EV costs in the three schemes are calculated on the real base loads. The total EV costs of the three schemes without renewable energy sources and battery lifetime cost reduction is shown in below table. 
Table .3: EV charging cost for three schemes without renewable energy sources and battery lifetime cost reduction.

\begin{tabular}{|l|l|c|}
\hline S. No & Schemes & $\begin{array}{l}\text { Charging cost of each scheme } \\
\text { in Rupees (₹) }\end{array}$ \\
\hline 1 & $\begin{array}{l}\text { Global optimal scheduling } \\
\text { scheme }\end{array}$ & 237.26 \\
\hline 2 & $\begin{array}{l}\text { Local optimal scheduling } \\
\text { scheme }\end{array}$ & 240.52 \\
\hline 3 & Equal allocation scheme & 261.88 \\
\hline
\end{tabular}

After including the battery lifetime cost reduction in the total costs for the two schemes, the charging cost of each scheme is shown in below table.

Table. 4: EV charging cost for three schemes with battery lifetime cost reduction and without renewable energy sources.

\begin{tabular}{|l|l|l|}
\hline S. No & Schemes & $\begin{array}{l}\text { Charging cost of each scheme } \\
\text { in Rupees (₹) }\end{array}$ \\
\hline 1 & $\begin{array}{l}\text { Global optimal scheduling } \\
\text { scheme }\end{array}$ & 244.57 \\
\hline 2 & $\begin{array}{l}\text { Local optimal scheduling } \\
\text { scheme }\end{array}$ & 246.24 \\
\hline
\end{tabular}

After including renewable energy sources cost in the total costs of the three schemes, the charging cost of each scheme is shown in below table.

Table .5: EV charging cost for three schemes with both battery lifetime cost reduction and renewable energy sources.

\begin{tabular}{|l|l|l|}
\hline S. No & Schemes & $\begin{array}{l}\text { Charging cost of each scheme } \\
\text { in Rupees (₹) }\end{array}$ \\
\hline 1 & $\begin{array}{l}\text { Global optimal scheduling } \\
\text { scheme }\end{array}$ & 165.17 \\
\hline 2 & $\begin{array}{l}\text { Local optimal scheduling } \\
\text { scheme }\end{array}$ & 166.84 \\
\hline 3 & Equal allocation scheme & 182.48 \\
\hline
\end{tabular}

To compare these three schemes including renewable energy sources and battery lifetime cost reduction is contribute decrease the price to the customers. The main reason is to renewable energy sources plays major role in EV charging cost minimization. After introducing renewable sources the costs in global, local and equal allocation scheme are nearly $30 \%$ decrease its original electric vehicle cost per day. It's more benefit for EV customer as well as grid point of view. If any peak loading conditions renewable energy sources supply the power to grid.

\section{Conclusion}

The optimal scheduling problem for Electric Vehicle charging/discharging by deliberates the battery lifetime cost reduction and renewable energy sources (wind and solar). To compare this three modes of operation in the optimal scheduling problem, to optimize the total cost of the EV's charging. The Global optimal scheduling scheme gives the optimal cost of EV charging compared to local and equal allocation scheme. But the global optimal scheme has impractical, Because of all the EVs are in the same time of charging then its face overloading problem. To neglect this problem local optimal scheduling problem is more effective in this for overloading problem and EV arrival time also considered as per sliding window. The local optimal scheduling problem also to optimize the total EV's cost in the existing ongoing EV set in the sub-group. In a large population of EV also it is hardy to the dynamic EV arrivals. The simulation outcomes of EV proven that the overall cost of EV's are decreased and the performance of local optimal scheduling cost is closest compared to the global optimal scheduling.

\section{References}

[1] "The Next Generation of Hybrid cars: plug-in Hybrids can help reduce global warming and slash oil dependency," N.R.D. council, Ed., ed, pp.4, July 2007.

[2] Mehdi Etezadi-Amoli, K choma, and J. Stefani, "Rapid charge electric vehicle stations," IEEE Transactions on power delivery, Vol. 25, pp. 1883-1887, 2010.

[3] International Energy Agency (IEA) and the Electric Vehicles Initiative of the clean energy ministerial (EVI), "global ev outlook: Understanding the Electric Vehicle landscape to 2020," April 2013.

[4] C. H. Stephan and J. Sullivan, "Environmental and energy implications of plug-in electric vehicles," Environmental science and technology, Vol.42, pp.1185-1190, 2008.

[5] K. Young, C. Wang and K. Strunz, "Electric vehicle battery technologies," in electric vehicle integration into modern power networks, ed: Springer, 2013, pp. 15-56.

[6] A. Maitra, J. Taylor, D. Brooks, M. Alexander and M. Duvall, "Integrating plug-in electric vehicles with the distribution system," in $20^{\text {th }}$ International Conference and Exhibition on Electricity Distribution, CIRED 2009, June 8, 2009- June 11, 2009, prague, Czech republic, 2009.

[7] C. Liu, K. T. Chau, D. Wu, and S. Gao, "Opportunities and challenges of vehicle-to-home, vehicle-to-vehicle and vehicle-to-grid technologies," Proc. of IEEE, Vol. 101, No. 11, pp. 2409-2427, November 2013.

[8] B. Lunza, Z. Yana, J.B. Gerschlerb and D. Sauera, "Review of the impact of vehicle-to-grid technologies on distribution systems and utility interfaces," Energy Policy Journal, Vol. 46, pp. 511-519, July 2012.

[9] Yifeng He, Bala Venkatesh, and Ling Guan, "Optimal scheduling for charging and discharging of electric vehicles", IEEE Transactions on Smart Grid, Vol. 3, No. 3, pp. 1095-1105, Sep. 2012.

[10] J. H. Chow, F. F. Wu, and J. A. Momoh, “Applied Mathematics for Restructured Electric Power Systems: Optimization, Control, and Computational Intelligence", 1st ed. New York: Springer, Nov. 2004.

[11] S. Boyd and L. Vandenberghe, Convex Optimization. Cambridge, U.K.: Cambridge Univ. Press, 2004.

[12] M. Grant and S. Boyd, "Graph implementations for nonsmooth convex programs," in Recent Advances in Learning and Control. NewYork: Springer, pp. 95-110, 2008.

[13] Tohid Shekari, Sajjad Golshannavaz, Farrokh Aminifar, "Techno-economic collaboration of PEV fleets in energy management of microgrids", IEEE Transactions on power system, Vol. 32,No. 5, pp. 3833-3841, Sep 2017.

[14] Norberto Fueyo, YosuneSanz, Marcos Rodrigues, Carlos Montanes, Cesar Dopazo, "The use of cost-generation curves for the analysis of wind electricity costs in Spain", ELSEVIER, Applied Energy.

[15] P. S.Moses, S. Deilami, A. S.Masoum, andM. A. S. Masoum, "Power quality of smart grids with plug-in electric vehicles considering battery charging profile," in Proc. 2010 IEEE PES Innov. Smart Grid Technol. Conf. Eur., pp. 1-7, Oct. 2010

[16] W. Shi, X. Xie, C.-C. Chu, and R. Gadh, "Distributed optimal energy management in microgrids," IEEE Transactions on Smart Grid, Vol. 6, No. 3, pp. 1137-1146, May 2015.

[17] X. Luo, S. W. Xia, and K. W. Chan, "A simple decentralized charging control scheme of plug-in electric vehicles for alleviating wind farm intermittency," Energy Procedia, Vol. 61, pp. 1789-1792, Dec. 2014.

[18] L. Deng, B. F. Hobbs and P. Renson, "What is the cost of negative bidding by wind? A unit commitment analysis of cost and emissions," IEEE Trans. Power Syst., Vol. 30, No. 4, pp. 18051814, Jul. 2015.

[19] P. Vithayasrichareon and I. F. MacGill, "Impacts of generationcycling costs on future electricity generation portfolio investment," in Proc. EEE PES General Meeting, National Harbor, MD, USA, 2014, pp. 1-5.

[20] C. Guille and G. Gross, "A conceptual framework for the vehicle- to- grid (V2G) implementation," Energy Policy, Vol. 37, No. 11, pp. 4379-4390, Nov. 2009.

[21] T. Bharath Kumar, O. Chandra Sekhar, M. Ramamoorty, S.V. N.L. Lalitha, "Evaluation of power capacity availability at load bus in a composite power system", IEEE J. Emerg. Sel. Top. Power Electron. 4 (4) (2016) 1324-1331. 
[22] T. Bharath Kumar, O. Chandra Sekhar, M. Ramamoorty, "Composite power system reliability evaluation using modified minimal cut set approach", Alexandria Engineering Journal - Elsevier, 2017 (Accepted- Early access). https://doi.org/10.1016/j.aej.2017.09.008

[23] J. García-Villalobos, I. Zamora, J. I. San Martín, F. J. Asensio, and V. Aperribay, "Plug-in electric vehicles in electric distribution networks: A review of smart charging approaches," Renew able and Sustainable Energy Reviews, Vol. 38, pp. 717-731, Oct. 2014

[24] F. R. Islam and H. R. Pota, "Integrating smart PHEVs in future smart grid," in Renewable Energy Integration, pp. 239-258, Springer Singapore, 2014.

[25] P. Sadeghi-Barzani, A. Rajabi-Ghananieh, and H. KazemiKaregar, "Optimal fast charging station placing and sizing," Appl. Energy, Vol. 125, pp. 289-299, Jul. 2014.

[26] Y. Zheng, Z. Y. Dong, Y. Xu, K. Meng, J. H. Zhao, and J. Qiu, "Electric vehicle battery charging swap stations in distribution systems: comparison study and optimal planning," IEEE Trans. Power Syst., Vol. 29, No. 1, pp. 221-229, Jan. 2014.

[27] M.F. Shaaban, M. Ismail, E.F. El-Saadany, W. Zhuang, "Real time PEV charging/discharging coordination in smart distribution systems", IEEE Transactions on Smart Grid, Vol.5, No.4, pp.1797-1807, July 2014

[28] The Clean-tech Market Authority. (May 2013, July 2013).PlugIn Vehicle Sales Hit 100,000 in Past Two Years available http://www.cleanedge.com/Resources/news/Plug-In- VehicleSales-Hit-100\%2C000-in-Past-Two-Years.

[29] http://www.livemint.com/Opinion/77tJwKaGstCLLjP9etjTFJ/Gl obal-electric-vehicle-revolution-by 2020.html.

[30] Website for Toronto Hydro-Electric System [Online]. Available: http://www.torontohydro.com/

[31] Chevy Volt Website [Online]. Available: http://www.chevyvolt. Net/chevrolet-volt-specs.html.

[32] M. Grant and S. Boyd, CVX: Matlab Software for Disciplined Convex Programming [Online]. Available: http://cvxr.com/cvx Dec. 2010, version 1.21

[33] Ramkiran, D.S., Madhav, B.T.P., Prasanth, A.M., Harsha, N.S. Vardhan, V., Avinash, K., Chaitanya, M.N. And Nagasai, U.S., 2015. Novel Compact Asymmetrical Fractal Aperture Notch Band Antenna. Leonardo Electronic Journal Of Practices And Technologies, 14(27), Pp. 1-12.

[34] Jawahar, A. And Koteswara Rao, S., 2015. Recursive Multistage Estimator For Bearings Only Passive Target Tracking In Esm Ew Systems. Indian Journal Of Science And Technology, 8(26),

[35] Kishore, P.V.V., Sastry, A.S.C.S. And Kartheek, A., 2014. Visual-Verbal Machine Interpreter For Sign Language Recognition Under Versatile Video Backgrounds, 1st International Conference On Networks And Soft Computing, Icnsc 2014 - Proceedings 2014, Pp. 135-140.

[36] Sarma, G.S., Asadi, S.S. And Narayana, S.L., 2016. Creation Of Web Based Decision Support Information System For Evaluation Of Topographic Characteristics Using Remote Sensing \& Gis And Visual Basic Programe. International Journal Of Civi Engineering And Technology, 7(6), Pp. 621-634.

[37] Aditya Varma, K.V., Manideep, T. And Asadi, S.S., 2016. A Critical Comparison Of Quantity Estimation For Gated Community Construction Project Using Traditional Method Vs Plan Swift Software: A Case Study. International Journal Of Civil Engineering And Technology, 7(6), Pp. 707-713.

[38] Karthik, G.V.S., Fathima, S.Y., Rahman, M.Z.U., Ahamed, S.R And Lay-Ekuakille, A., 2013. Efficient Signal Conditioning Techniques For Brain Activity In Remote Health Monitoring Network. Ieee Sensors Journal, 13(9), Pp. 3273-3283. 